\title{
Infinitely many states and stochastic symmetry in a Gaussian Potts-Hopfield model
}

\author{
A.C.D. van Enter and H.G. Schaap*
}

November 5, 2018

\section{Introduction}

In this paper we study the mean-field Potts model with Hopfield-Mattis disorder, in particular with Gaussianly distributed disorder. This model is a generalization of the model studied in BvEN. It provides yet another example of a disordered model with infinitely many low-temperature pure states, such as is sometimes believed to be typical for spin-glasses [MPV]. In our model, however, in contrast to [BvEN], instead of chaotic pairs we find that the chaotic size dependence is realized by chaotic $q(q-1)$-tuples. For the notion of chaotic size dependence, and the notion of chaotic pairs which were introduced by Newman and Stein we refer to $\mathbb{N S}$, NS2 and references mentioned there. Compare also [BvEN] and $[\mathrm{Ni}]$. For an extensive discussion of the Hopfield model, including some history and its relation with the theory of neural networks, see [B, pag. 133 and further] or [BG]. A somewhat different generalization of the Hopfield model to Potts spins can be found in $[G]$.

We are concerned in particular with the infinite-volume limit behaviour of the Gibbs and ground state measures. The possible limit points are labeled as the minima of an appropriate mean-field (free) energy functional. These minima can be obtained as solutions of a suitable mean-field equation. These minima lie on the minimal-free-energy surface, which is a $m(q-1)$-sphere in

*Instute for Theoretical Physics, Rijksuniversiteit Groningen, NL-9747 AG Groningen, The Netherlands; e-mail: A.C.D.van.Enter@phys.rug.nl, H.G.Schaap@phys.rug.nl 
the $\left(\mathbf{e}^{1}, \cdots, \mathbf{e}^{q}\right)^{\otimes m}$ space. This space for $q$-state Potts spins and $m$ patterns is formed by the m-fold product of the hyperplane spanned by the end points of the unit vectors $\mathbf{e}_{q}$, which are the possible values of the spins. But only a limited area of the minimal free energy surface is accessible. Only those values for which certain mean-field equations hold, are allowed. These equations have the structure of fixed point equations. We derive them in chapter

4. To obtain the Gibbs states we need to find the solutions of these equations on the minimal free energy surface.

The structure of the ground or Gibbs states for $q=2$ and $\xi^{k}$ Gaussian with $m=2$ is known since a few years BvEN. Due to the Gaussian distribution we have a nice symmetric structure: the ground states form a circle. For a fixed configuration and a large finite volume the possible order-parameter values become close to two diametrical points (which ones depend on the volume of the system) on this circle. This paper treats the generalization of this structure to $q$-state Potts spins with $q>2$. To have a concrete example, we concentrate on the case $q=3$. It turns out that we again obtain a circle symmetry but also a discrete symmetry, which generalizes the one for Ising spins. One gets instead of a single pair a triple of pairs (living on 3 separate circles), where for each pair one has a similar structure as for the single pair

for $q=2$. For $q>3$ we get $\frac{q(q-1)}{2}$ pairs and a similar higher-dimensional structure.

Our model displays quenched disorder. This means that we look at a fixed, particular realization of the patterns. It turns out that there is some kind of self-averaging. The thermodynamic behaviour of the Hamiltonian is the same for almost every realization. This is the case for the free energy and the associated fixed point equations, as is familiar from many quenched disordered models. However, this is not precisely true for the order parameters. We will see that they show a form of chaotic size dependence, i.e. the behaviour strongly depends both on the chosen configuration and on the way one takes the infinite-volume limit $N \rightarrow \infty$ (that is, along which subsequence).

\section{Notations and definitions}

We start with some definitions. Consider the set $\Lambda_{N}=\{1, \cdots, N\} \in \mathbf{N}^{+}$. Let the single-spin space $\chi$ be a finite set and the $\mathrm{N}$-spin configuration space 
be $\chi^{\otimes N}$. We denote a spin configuration by $\sigma$ and its value at site i by $\sigma_{i}$. We will consider Potts spins, in the $\mathrm{Wu}$ representation [Wu]. The set $\chi^{\otimes N}$ is then the $N$-fold tensor product of the set $\chi=\left\{\mathbf{e}^{1}, \cdots, \mathbf{e}^{q}\right\}$. The $\mathbf{e}^{\sigma}$ are the projection of the spinvectors $\mathbf{e}_{\sigma}$ on the hypertetrahedron in $\mathbf{R}^{q-1}$ spanned by the end points of $\mathbf{e}_{\sigma}$. For $q=3$ we get for example for $\mathbf{e}^{1}, \mathbf{e}^{2}$ and $\mathbf{e}^{3}$ the vectors:

$$
\left\{\left(\begin{array}{l}
1 \\
0
\end{array}\right),\left(\begin{array}{c}
-\frac{1}{2} \\
\frac{1}{2} \sqrt{3}
\end{array}\right),\left(\begin{array}{c}
-\frac{1}{2} \\
-\frac{1}{2} \sqrt{3}
\end{array}\right)\right\} .
$$

The Hamiltonian of our model is defined as follows:

$$
\begin{gathered}
-\beta H_{N}=\frac{\beta}{N} \sum_{k=1}^{m} \sum_{i, j=1}^{N} \xi_{i}^{k} \xi_{j}^{k} \delta\left(\sigma_{i}, \sigma_{j}\right), \text { with } \\
\delta\left(\sigma_{i}, \sigma_{j}\right)=\frac{1}{q}\left[1+(q-1) \mathbf{e}^{\sigma_{i}} \cdot \mathbf{e}^{\sigma_{j}}\right],
\end{gathered}
$$

where $\xi_{i}^{k}$ is the $i$-th component of the random $N$-component vector $\xi^{k}$. For the $\xi_{i}^{k}$ 's we choose i.i.d. $N(0,1)$ distributions. The vectors $\xi^{k}=\left(\xi_{1}^{k}, \cdots, \xi_{N}^{k}\right)$, by analogy with the standard Hopfield model, are called patterns. If we combine the above, we can rewrite the Hamiltonian $H_{N}$ as:

$$
-\beta H_{N}=\beta \frac{q-1}{q} N \sum_{k=1}^{m}\left[\left(\frac{\sum \xi_{i}^{k} \mathbf{e}^{\sigma_{i}}}{N}\right)^{2}+\frac{1}{q-1}\left(\frac{\sum \xi_{i}^{k}}{N}\right)^{2}\right] .
$$

So asymptotically

$$
-\beta H_{N}=N \frac{K}{2} \sum_{k=1}^{m} q_{k N}^{2}
$$

$$
\text { with } K=2 \beta\left(\frac{q-1}{q}\right) \text { and order parameters } q_{k N}=\frac{1}{N} \sum_{i=1}^{N} \xi_{i}^{k} \mathbf{e}^{\sigma_{i}} .
$$

The last term is an irrelevant constant; in fact it approaches zero, due to the strong law of large numbers. (The $\xi_{i}^{k}$ 's are i.i.d. $N(0,1)$ distributed so $E \xi_{i}^{k}=0$.) Note that any i.i.d. distribution with zero mean, finite variance and symmetrically distributed around zero will give an analogous form of $H_{N}$, but we plan to consider only Gaussian distributions, for which we find that a continous symmetry can be stochastically broken, just as in BvEN. From now on we drop the subscript $N$ to simplify the notation, when no confusion can arise. 
Furthermore we introduce two representations for the order parameters $\vec{q}$. If we assume $m=2$ then $\vec{q}=\left(\vec{q}_{1}, \vec{q}_{2}\right)$ and the definitions are as follows: if we consider the space $\mathbf{R}^{\mathbf{q}-\mathbf{1}}$ spanned by the vectors $\mathbf{e}^{1}, \cdots, \mathbf{e}^{q}$, the $\vec{x}$-plane, we define $\vec{q}=\left(x_{1}, \cdots, x_{2(q-1)}\right)$. It is often more convenient to look at the (higherdimensional) $\left(\mathbf{e}_{1}, \cdots, \mathbf{e}_{q}\right)$-space. In that case we take $\vec{q}=\left(a_{1}, a_{2}, a_{3}, b_{1}, b_{2}, b_{3}\right)$ for $q=3$ and an equivalent equation for other values of $q$. For $m \neq 2$ the definitions are analogous.

\section{Ground states}

Now it is time to reveal the characteristics of the ground states for the Potts model. First we discuss the simple behaviour for 1 pattern. Then the more interesting part: $q>2$ and 2 patterns.

\subsection{Ground states for 1 pattern}

For one pattern $\xi$ the Hamiltonian is of the following form:

$$
-\beta H=N \frac{K}{2} \vec{q}_{1}^{2}=\frac{\beta}{N} \sum_{i, j=1}^{N} \xi_{i} \xi_{j} \delta\left(\sigma_{i}, \sigma_{j}\right) .
$$

We easily see that the ground states are obtained by directing the spins with $\xi_{i}>0$ in one direction and the spins with $\xi_{i} \leq 0$ in a different direction. If we have as the distribution for the $\xi_{i}$ 's $P\left(\xi_{i}= \pm 1\right)=\frac{1}{2}$, then the order parameter is of the form: $\vec{q}_{1}=\frac{1}{2}\left(\mathbf{e}^{\sigma_{i}}-\mathbf{e}^{\sigma_{j}}\right)$, with $1 \leq i, j \leq q$ and $i \neq j$, see also vEHP. So for $q=3$ we have only 6 ground states. They form a regular hexagon: $\left( \pm \frac{3}{4}, \mp \frac{\sqrt{3}}{4}\right), \pm\left(\frac{3}{4}, \frac{\sqrt{3}}{4}\right),\left(0, \pm \frac{\sqrt{3}}{2}\right)$. This regular hexagon with its interior is the convex set of possible order parameter values. It is easy to see that for $\xi_{i} N(0,1)$-distributed we get the same ground states except for a scaling factor $\sqrt{2 / \pi}$ multiplying the values of the order parameter values.

\subsection{Ground states for 2 patterns}

The Hamiltonian for 2 patterns (Gaussian i.i.d.) is:

$$
-\beta H_{N}=\frac{\beta}{N} \sum_{i, j=1}^{N}\left(\xi_{i} \xi_{j}+\eta_{i} \eta_{j}\right) \delta\left(\sigma_{i}, \sigma_{j}\right)=N \frac{K}{2}\left(\vec{q}_{1}^{2}+\vec{q}_{2}^{2}\right) .
$$


Similarly as in [BvEN], we make use of the fact that the distribution of 2 independent identically distributed Gaussians has a continous rotation symmetry. This symmetry shows also up in the order parameters. Let

$$
\vec{q}_{1}(\theta)=\left(\begin{array}{c}
x_{1}(\theta) \\
x_{2}(\theta)
\end{array}\right)=\left(\begin{array}{c}
\alpha \sin \theta \\
\beta \sin \theta
\end{array}\right), \vec{q}_{2}(\theta)=\left(\begin{array}{c}
x_{3}(\theta) \\
x_{4}(\theta)
\end{array}\right)=\left(\begin{array}{c}
\alpha \cos \theta \\
\beta \cos \theta
\end{array}\right)
$$

with $(\alpha, \beta)$ a ground state associated to the special case $\theta=0$ i.e. to the second pattern. We note that asymptotically for large $N$ we get the same ground state energy per site for each value of $\theta$. Because the surface on which the Hamiltonian is constant is of the form $\vec{q}_{1}^{2}+\vec{q}_{2}^{2}=C^{2}$, these are the only ground states. For finite $N$, however, there are finitely many $(q(q-1))$ ground states, corresponding to one particular value of $\theta$ (The exact symmetry of choosing a different pair of Potts directions gives the $q(q-1)$ ground states). This is an example of chaotic size dependence, based on the breaking of a stochastic symmetry, of the same nature as in [BvEN]. Because of weak compactness, different subsequences exist whose $q(q-1)$-tuples of ground states converge to $q(q-1)$-tuples, associated to particular $\theta$-values. These subsequences depend on the random pattern realization. See Appendix A. For further background on chaotic size dependence and its role in the theory of metastates we refer to [NS]. For $m \geq 3$ patterns one has the same discrete structure as before, but instead of a continous circle symmetry we have a continous $m$-sphere symmetry (isomorpic to $O(m)$ ).

\section{Positive temperatures}

At positive temperatures instead of minimizing an energy one needs to minimize a free energy expression.

By making use of arguments from large deviation theory we obtain (see e.g. [HvEQ]):

$$
-\beta f(\beta)=\sup _{\vec{q}_{1}, \vec{q}_{2}}\left\{Q\left(\vec{q}_{1}, \vec{q}_{2}\right)-c^{\star}\left(\vec{q}_{1}, \vec{q}_{2}\right)\right\},
$$

where $f$ is the free energy per spin and $-\beta H=N \frac{K}{2}\left(\vec{q}_{1}^{2}+\vec{q}_{2}^{2}\right) \equiv N Q$. The function $c^{\star}$ is the Legendre transform of $c$, where $c$ is defined as follows:

$$
c(\vec{t})=\lim _{N \rightarrow \infty} \frac{1}{N} \ln \left\{\mathbf{E}_{\sigma} \exp \left(\overrightarrow{t_{1}} \cdot N \vec{q}_{1}+\vec{t}_{2} \cdot N \vec{q}_{2}\right)\right\} .
$$


Here $\vec{t}_{1}$ and $\vec{t}_{2}$ are vectors in $\mathbf{R}^{q-1}$ and $\operatorname{tr}_{\sigma}$ is the normalised trace at a single site. To determine the supremum (maximum) we differentiate and put the derivative equal to 0 . This implies that for $\overrightarrow{q_{1}}$ and $\overrightarrow{q_{2}}$ it holds:

$$
\begin{gathered}
\left(\vec{q}_{1}, \vec{q}_{2}\right) \max =\nabla c\left(\nabla Q\left(\vec{q}_{1}, \vec{q}_{2}\right)\right)=\nabla c\left(K \vec{q}_{1}, K \vec{q}_{2}\right), \text { with } \\
\left\{\begin{array}{l}
K \vec{q}_{1}=\frac{\partial Q}{\partial \vec{q}_{1}} \\
K \overrightarrow{q_{2}}=\frac{\partial Q}{\partial \vec{q}_{2}} .
\end{array}\right.
\end{gathered}
$$

We make use of the fact that for a convex function $c, \nabla c^{\star}=(\nabla c)^{-1}$ (see also [BG, chap. 3] and compare pag. 27). Now let us rewrite $c(\vec{t})$ :

$$
\begin{gathered}
c(\vec{t})=\lim _{N \rightarrow \infty} \frac{1}{N} \ln \left\{\mathbf{E}_{\sigma} \exp \left(\overrightarrow{t_{1}} \cdot N \overrightarrow{q_{1}}+\overrightarrow{t_{2}} \cdot N \overrightarrow{q_{2}}\right)\right\}= \\
\cdots=<\ln \operatorname{tr}_{\sigma}\left\{\exp \left(\xi \overrightarrow{t_{1}}+\eta \overrightarrow{t_{2}}\right) \cdot \mathbf{e}^{\sigma}\right\}>_{\xi, \eta} .
\end{gathered}
$$

Plugging this into (3) we get the mean field equations for the order parameters which have the structure of a system of fixed point equations $\vec{q}=F(\vec{q})$ :

$$
\left\{\begin{array}{l}
\vec{q}_{1}=\left\langle\frac{\operatorname{tr}_{\sigma}\left\{\xi \mathbf{e}^{\sigma} \exp \left[K\left(\xi q_{1}+\eta q_{2}\right) \cdot \mathbf{e}^{\sigma}\right]\right\}}{\operatorname{tr}_{\sigma}\left\{\exp \left[K\left(\xi q_{1}+\eta q_{2}\right) \cdot \mathbf{e}^{\sigma}\right]\right\}}\right\rangle_{\xi, \eta} \\
\vec{q}_{2}=\left\langle\frac{\operatorname{tr}_{\sigma}\left\{\eta \mathbf{e}^{\sigma}\left\{\exp \left[K\left(\xi q_{1}+\eta q_{2}\right) \cdot \mathbf{e}^{\sigma}\right]\right\}\right.}{\operatorname{tr}_{\sigma}\left\{\exp \left[K\left(\xi q_{1}+\eta q_{2}\right) \cdot \mathbf{e}^{\sigma}\right]\right\}}\right\rangle_{\xi, \eta} .
\end{array}\right.
$$

If we are in the allowed area, that is, the domain of definition of $\mathrm{F}$, it is equivalent to look in the $\left(\mathbf{e}_{1}, \cdots, \mathbf{e}_{q}\right)$-space. We may rewrite (雨) in this area as follows:

$$
\left\{\begin{array}{c}
\vec{q}_{1}=\left(\begin{array}{c}
a_{1} \\
\vdots \\
a_{q}
\end{array}\right)=\left(\begin{array}{c}
\left\langle\frac{\xi \exp K\left(\xi a_{1}+\eta b_{1}\right)}{\sum_{i=1}^{q} \exp K\left(\xi a_{i}+\eta b_{i}\right)}\right\rangle_{\xi, \eta} \\
\vdots \\
\left\langle\frac{\xi \exp K\left(\xi a_{q}+\eta b_{q}\right)}{\sum_{i=1}^{q} \exp K\left(\xi a_{i}+\eta b_{i}\right)}\right\rangle_{\xi, \eta}
\end{array}\right) \\
\vec{q}_{2}=\left(\begin{array}{c}
b_{1} \\
\vdots \\
b_{q}
\end{array}\right)=\left(\begin{array}{c}
\left\langle\frac{\eta \exp K\left(\xi a_{1}+\eta b_{1}\right)}{\sum_{i=1}^{q} \exp K\left(\xi a_{i}+\eta b_{i}\right)}\right\rangle_{\xi, \eta} \\
\vdots \\
\left\langle\frac{\eta \exp K\left(\xi a_{q}+\eta b_{q}\right)}{\sum_{i=1}^{q} \exp K\left(\xi a_{i}+\eta b_{i}\right)}\right\rangle_{\xi, \eta}
\end{array}\right)
\end{array}\right.
$$

with $\vec{q}_{1}=\sum_{i=1}^{q} a_{i} \mathbf{e}_{i}$ and $\vec{q}_{2}=\sum_{i=1}^{q} b_{i} \mathbf{e}_{i}$. 


\subsection{Ising spins}

If we look at the behaviour for $N \rightarrow \infty$, then due to the strong law of large numbers $\frac{1}{N} \sum_{i=1}^{N} \xi_{i}=\mathbf{E} \xi=0$. Each coordinate $a_{j}$ of vector $\vec{q}_{1}=\left(a_{1}, a_{2}\right)$ is defined as $\frac{1}{N} \sum_{i=1}^{N} \xi_{i} \delta\left(\sigma_{i}, \sigma_{j}\right)$. This means that $a_{j}$ is the contribution of the spins in the $j$-th direction to the sum $\frac{1}{N} \sum_{i=1}^{N} \xi_{i}$. Therefore: $a_{1}+a_{2}=$ $\frac{1}{N} \sum_{i=1}^{N} \xi_{i}=0$ a.e. This gives a necessary condition for the allowed area of Ising spins:

$$
a_{1}=-a_{2} \wedge b_{1}=-b_{2} .
$$

Furthermore for all Gibbs states the value of the energy is constant, therefore:

$$
a_{1}^{2}+a_{2}^{2}+b_{1}^{2}+b_{2}^{2}=\frac{r^{\star 2}}{2} .
$$

When we substitute (5) in equation (6) and project the result to the $\left(x_{1}, x_{2}\right)$ plane by the projection $\Pi: \mathbf{e}_{1} \rightarrow 1, \mathbf{e}_{2} \rightarrow-1$, we obtain the following equation:

$$
x_{1}^{2}+x_{2}^{2}=r^{\star 2} \text {. }
$$

Thus to get the radius of the circle of Gibbs states $r^{\star}$, just take the point $\overrightarrow{q_{1}}=(a,-a), \overrightarrow{q_{2}}=(0,0)$. This corresponds to the point $(2 a, 0)$ in the $\left(x_{1}, x_{2}\right)-$ plane, by the projection $\Pi$. Of course $2 a=r^{\star}$.

With this we calculate the equation for the first coordinate of $\vec{q}_{1}$ in the $\left(\mathbf{e}_{1}, \mathbf{e}_{2}\right)$-plane by substituting the corresponding fixed point equation:

$$
\begin{gathered}
a=\frac{1}{2 \pi} \iint \xi \frac{\exp \beta \xi a}{\exp \beta \xi a+\exp (-\beta \xi a)} \exp \left(-\frac{\xi^{2}+\eta^{2}}{2}\right) d \xi d \eta= \\
\frac{1}{\sqrt{2 \pi}} \int \xi \frac{\exp \beta \xi a}{\exp \beta \xi a+\exp (-\beta \xi a)} \exp \left(-\frac{\xi^{2}}{2}\right) d \xi .
\end{gathered}
$$

We replaced $K$ by $\beta$, because for Ising spins $K=2 \beta(2-1) / 2=\beta$. The equation for the second coordinate of $\vec{q}_{1}$ we calculate in the same way. The vector $\vec{q}_{2}$ is simply $(0,0)$. Now project $\vec{q}_{1}$ and $\vec{q}_{2}$ to the $\left(x_{1}, x_{2}\right)$-plane. That is done by subtracting the second coordinate of the $\vec{q}_{i}$ 's from the first one. We get the following equation for the radius $r^{\star}$ :

$$
r^{\star}=\frac{1}{\sqrt{2 \pi}} \int \xi \tanh \left(\frac{\beta \xi r^{\star}}{2}\right) \exp \left(-\frac{\xi^{2}}{2}\right) d \xi .
$$


For $\beta>\beta_{0}$ this equation has a nontrivial solution for $r^{\star}$. The equation is the same as in $B v E N$ except the factor $1 / 2$ in the tanh. This is due to our using the $\mathrm{Wu}$ representation.

\subsection{Potts spins}

If we take $q=3$, then $K=\frac{4}{3} \beta$. The set of ground states now can be parametrized by three (in general $\frac{q(q-1)}{2}$ ) circles, and similarly for the lowtemperature Gibbs states. To obtain the radius $\hat{r}$ of such a circle parametrizing the ground or Gibbs states, we follow the same recipe as in the case of Ising spins. Here we take the point $\left(\vec{q}_{1}, \vec{q}_{2}\right)$ with $\vec{q}_{1}=(0, \hat{r} / \sqrt{3},-\hat{r} / \sqrt{3})$ and $\overrightarrow{q_{2}}=(0,0,0)$ (the representatives of both $\vec{q}_{i}$ in the $\left(\mathbf{e}_{1}, \mathbf{e}_{2}, \mathbf{e}_{3}\right)$-plane). Now $\vec{q}_{1}$ projects to $(0, \hat{r})$ by the projection:

$$
\left(\begin{array}{l}
x_{1} \\
x_{2}
\end{array}\right)=\left(\begin{array}{rrr}
1 & -\frac{1}{2} & -\frac{1}{2} \\
0 & \frac{1}{2} \sqrt{3} & -\frac{1}{2} \sqrt{3}
\end{array}\right)\left(\begin{array}{l}
a_{1} \\
a_{2} \\
a_{3}
\end{array}\right) .
$$

So if we substitute the corresponding fixed point equations for $\vec{q}_{1}$ in the $\left(\mathbf{e}_{1}, \mathbf{e}_{2}, \mathbf{e}_{3}\right)$-plane, we get for the order parameter values $\left(a_{1}, a_{2}, a_{3}\right) \equiv \vec{q}_{1}$ the following mean field equations:

$$
\left(\begin{array}{l}
a_{1} \\
a_{2} \\
a_{3}
\end{array}\right)=\left(\begin{array}{c}
0 \\
\frac{1}{\sqrt{2 \pi}} \int \xi \frac{\exp (K \xi \hat{r} / \sqrt{3})}{\exp (K \xi \hat{r} / \sqrt{3})+\exp (-K \xi \hat{r} / \sqrt{3})+1} \exp \left(-\frac{\xi^{2}}{2}\right) d \xi \\
\frac{1}{\sqrt{2 \pi}} \int \xi \frac{\exp (-K \xi \hat{r} / \sqrt{3})}{\exp (K \xi \hat{r} / \sqrt{3})+\exp (-K \xi \hat{r} / \sqrt{3})+1} \exp \left(-\frac{\xi^{2}}{2}\right) d \xi
\end{array}\right) .
$$

Here $\left(a_{1}, a_{2}, a_{3}\right)=(0, \hat{r} / \sqrt{3},-\hat{r} / \sqrt{3})$. Thus by taking the difference between $a_{2}$ and $a_{3}$ and multiplying it by $\frac{1}{2} \sqrt{3}$, we finally get the following expression for the absolute value $\hat{r}$ :

$$
\begin{gathered}
\hat{r}=\frac{1}{2 \sqrt{\pi}} \sqrt{\frac{3}{2}} \int \xi \frac{\exp (K \xi \hat{r} / \sqrt{3})-\exp (-K \xi \hat{r} / \sqrt{3})}{\exp (K \xi \hat{r} / \sqrt{3})+\exp (-K \xi \hat{r} / \sqrt{3})+1} \exp \left(-\frac{\xi^{2}}{2}\right) d \xi= \\
\frac{1}{\sqrt{\pi}} \sqrt{\frac{3}{2}} \int \frac{\xi \sinh (K \xi \hat{r} / \sqrt{3})}{2 \cosh (K \xi \hat{r} / \sqrt{3})+1} \exp \left(-\frac{\xi^{2}}{2}\right) d \xi
\end{gathered}
$$

We can easily check that this expression indeed approaches the one for the radius for the circles through the ground states, by considering the behaviour of the integrand for $K \rightarrow \infty$. It behaves like:

$$
\int|\xi| \exp \left(-\frac{\xi^{2}}{2}\right) d \xi \text {. }
$$




\section{A Stochastic symmetry breaking for $q=3$}

In this Appendix we adapt the fluctuation analysis of BvEN to include Potts spins. We essentially follow the same line of argument, and find that the fluctuations, properly scaled, after dividing out the discrete symmetry, approach again a Gaussian process on the circle.

For notational simplicity we treat the case $q=3$ only. For $q>3$ a similar analysis applies. Define the function $\phi_{N, 2}$ as follows:

$$
\beta \phi_{N, 2}(\vec{z})=-Q(\vec{z})+\vec{z} \cdot \nabla Q(\vec{z})-c(\nabla Q(\vec{z})),
$$

where $c(\vec{t})$ equals:

$c(\vec{t})=\frac{1}{N} \ln \left\{\mathbf{E}_{\sigma} \exp \overrightarrow{t_{1}} \cdot N \overrightarrow{q_{1}}+\overrightarrow{t_{2}} \cdot N \overrightarrow{q_{2}}\right\}=\frac{1}{N} \sum_{i=1}^{N} \ln \left\{\mathbf{E}_{\sigma_{i}} \exp \overrightarrow{t_{1}} \cdot \xi_{i} \mathbf{e}^{\sigma_{i}}+\overrightarrow{t_{2}} \cdot \eta_{i} \mathbf{e}^{\sigma_{i}}\right\}$.

This $\phi_{N}$ is chosen such that for $N \rightarrow \infty$ the measure

$$
\tilde{\mathcal{L}}=\frac{e^{-\beta N \phi_{N}}}{Z_{N, \beta}} \rightarrow \mathcal{L}
$$

where $\mathcal{L}$ is the induced distribution of the overlap parameters.

For $q=3$ it holds:

$$
Q(\vec{z})=\frac{K}{2}\|\vec{z}\|_{2}^{2}=\frac{2}{3} \beta\|\vec{z}\|^{2}
$$

Thus:

$$
\begin{aligned}
\phi_{N, 2}(\vec{z})= & \frac{2}{3}\|\vec{z}\|_{2}^{2}-\frac{1}{\beta N} \ln \left\{\mathbf{E}_{\sigma} \exp \frac{4}{3} \beta\left(\xi_{i} \vec{z}_{1} \cdot \mathbf{e}^{\sigma_{i}}+\eta_{i} \vec{z}_{2} \cdot \mathbf{e}^{\sigma_{i}}\right)\right\} \equiv \frac{2}{3}\|\vec{z}\|_{2}^{2}-\frac{1}{\beta N} \Xi_{N, 2} . \\
\Xi_{N, 2}= & \sum_{i=1}^{N} \ln \left\{\frac{1}{3} \exp K\left(\xi_{i} z_{11}+\eta_{i} z_{21}\right)+\frac{2}{3} \exp -\frac{K}{2}\left(\xi_{i} z_{11}+\eta_{i} z_{21}\right) \cosh \frac{K \sqrt{3}}{2}\left(\xi_{i} z_{12}+\eta_{i} z_{22}\right)\right\}= \\
& \sum_{i=1}^{N} \ln \left\{\frac{1}{3} \phi_{1}\left(z_{11}, z_{22}\right)_{\xi, \eta}+\frac{2}{3 \sqrt{\phi_{1}\left(z_{11}, z_{22}\right)_{\xi, \eta}}} \phi_{2}\left(z_{12}, z_{22}\right)_{\xi, \eta}\right\} .
\end{aligned}
$$

Because for finite $N$ the set of 6 Gibbs states has a discrete symmetry, as mentioned before, we choose out of these 6 states one state we like, namely 
the one of the form $(0, \pm \alpha \sin \theta, 0, \pm \alpha \cos \theta)$. Note that the $\theta$ depends both on $N$ and on the realization of the random disorder variable. Then $z_{11}=$ $z_{21}=\phi_{1}=0$. Inserting this and defining $z_{12}=\tilde{z_{1}}$ and $z_{22}=\tilde{z_{2}}$ we get for $\phi$ :

$$
\phi\left(\tilde{z_{1}}, \tilde{z_{2}}\right)=\frac{2}{3}\left\|\left(\tilde{z_{1}}, \tilde{z_{2}}\right)\right\|_{2}^{2}-\frac{1}{\beta N} \sum_{i=1}^{N} \ln \left\{\frac{1}{3}+\frac{2}{3} \cosh \frac{2}{\sqrt{3}} \beta\left(\xi_{i} \tilde{z_{1}}+\eta_{i} \tilde{z_{2}}\right)\right\} .
$$

Putting $\left(z_{1}, z_{2}\right)=\frac{2}{\sqrt{3}}\left(\tilde{z_{1}}, \tilde{z}_{2}\right)$ we obtain:

$$
\phi\left(z_{1}, z_{2}\right)=\frac{1}{2}\left\|\left(z_{1}, z_{2}\right)\right\|_{2}^{2}-\frac{1}{\beta N} \sum_{i=1}^{N} \ln \left\{\frac{1}{2}+\cosh \beta\left(\xi_{i} z_{1}+\eta_{i} z_{2}\right)\right\}-\frac{1}{\beta N} \ln \frac{2}{3} .
$$

¿From now on the last term will be ignored. So it is enough to prove now that with the $\frac{1}{2}$ term we get the desired chaotic pairs structure between the patterns due to the quenched disorder for this class of ground states, once we divide out the appropriate discrete Potts permutation symmetry. Thus the original model displays chaotic 6-tuples.

Therefore we only need to control the fluctuations of $\phi$. Define

$f_{N}^{\star}(\vec{z})-\mathbf{E} f_{N}^{\star}(\vec{z}) \equiv \frac{1}{\beta N} \sum_{i=1}^{N} \ln \{\cosh \beta \vec{z} \cdot(\xi, \eta)\}-\frac{1}{\beta N} \sum_{i=1}^{N} \mathbf{E} \ln \{\cosh \beta \vec{z} \cdot(\xi, \eta)\}$.

This is the fluctuation of the Ising case which we can estimate by BvEN. Denote the corresponding $\phi$ function by $\phi^{\star}$. We start with the following lemma:

\section{Lemma A.1}

$$
\exp (-\beta N \phi) \leq \exp \left(-\beta N \phi^{\star}\right)
$$

Proof:

Because

$$
\exp (-\beta N \phi)=\exp \left(-\beta N \mathbf{E} \phi^{\star}\right) \exp \left(-\beta N\left(\phi-\mathbf{E} \phi^{\star}\right),\right.
$$

we only have to estimate the quantity $\phi-\mathbf{E} \phi^{\star}$. Notice that also a lower bound is essential, because the quantity can become negative. First the estimate from above:

$$
\phi-\mathbf{E} \phi^{\star}=\frac{1}{\beta N} \sum_{i=1}^{N} \ln \left\{\frac{1}{2}+\cosh \beta \vec{z} \cdot(\xi, \eta)\right\}-\frac{1}{\beta N} \sum_{i=1}^{N} \mathbf{E} \ln \{\cosh \beta \vec{z} \cdot(\xi, \eta)\} .
$$


Now use

$$
\begin{gathered}
\ln \left\{\frac{1}{2}+\cosh \beta \vec{z} \cdot(\xi, \eta)\right\}=\ln \left\{1+\frac{1}{2 \cosh \beta \vec{z} \cdot(\xi, \eta)}\right\}+\ln \{\cosh \beta \vec{z} \cdot(\xi, \eta)\} \leq \\
\ln \{\cosh \beta \vec{z} \cdot(\xi, \eta)\}+\ln \frac{3}{2} \text { to get } \\
\phi-\mathbf{E} \phi^{\star} \leq \frac{1}{\beta N} \sum_{i=1}^{N} \ln \{\cosh \beta \vec{z} \cdot(\xi, \eta)\}-\frac{1}{\beta N} \sum_{i=1}^{N} \mathbf{E} \ln \{\cosh \beta \vec{z} \cdot(\xi, \eta)\}+\frac{1}{\beta} \ln \frac{3}{2}= \\
f_{N}^{\star}(\vec{z})-\mathbf{E} f_{N}^{\star}(\vec{z})+\frac{1}{\beta} \ln \frac{3}{2} .
\end{gathered}
$$

This is because $\cosh x \geq 1$ for all $x \in \mathbf{R}$.

The lower bound is easy because:

$$
\frac{1}{\beta N} \sum_{i=1}^{N} \ln \left\{\frac{1}{2}+\cosh \beta \vec{z} \cdot(\xi, \eta)\right\} \geq \frac{1}{\beta N} \sum_{i=1}^{N} \ln \{\cosh \beta \vec{z} \cdot(\xi, \eta)\} .
$$

This is due to the fact that the function $\ln \alpha$ is monotonically increasing in $\alpha$. Then it follows that:

$$
\phi-\mathbf{E} \phi^{\star} \geq f_{N}^{\star}(\vec{z})-\mathbf{E} f_{N}^{\star}(\vec{z}) .
$$

Combine (11), (12) and use the fact that in the limit $\lim _{N \rightarrow \infty}$ the constant term $\frac{1}{\beta} \ln \frac{3}{2}$ does not contribute to the expression $\exp \left\{-\beta N\left(\phi-\mathbf{E} \phi^{\star}\right)\right\}$ to conclude the proof of lemma A.1.

Henceforth it is convenient to transform $\phi^{\star}$ to polar coordinates. Define $z=(r \cos \theta, r \sin \theta)$. Then (9) transforms to:

$$
\begin{gathered}
\left|\bar{f}_{N}^{\star}(r, \theta)\right|=\left|\frac{1}{\beta} \mathbf{E}_{\psi} \mathbf{E}_{\zeta} \ln \cosh \{\beta \zeta r \cos \psi\}-\frac{1}{\beta N} \sum_{i=1}^{N} \ln \cosh \left\{\beta r \zeta_{i} \cos \left(\theta-\psi_{i}\right)\right\}\right|= \\
\left|\mathbf{E} f_{N}^{\star}(r, \theta)-f_{N}^{\star}(r, \theta)\right| .
\end{gathered}
$$

Here $\zeta, \psi$ denote the polar decomposition of the two-dimensional vector $(\xi, \eta)$, i.e. $\zeta$ is distributed with density $x \exp -x^{2} / 2$ on $\mathbf{R}^{+}$and $\psi$ uniformly on the circle $[0,2 \pi)$. See [BvEN, page 188]. This we see easily because:

$\xi z_{1}+\eta z_{2}=(\zeta \cos \psi)(r \cos \theta)+(\zeta \sin \psi)(r \sin \theta)=\zeta r(\cos \theta \cos \psi+\sin \theta \sin \psi)=$ 


$$
\zeta r \cos (\theta-\psi) \text { and } \mathbf{E}_{\psi} \cos (\theta-\psi)=\mathbf{E}_{\psi} \cos \psi .
$$

With $\phi^{\star}$ in this form, estimate (10) of lemma A.1 is not very useful, since the fluctuations of $\phi$ reach their minimum for a different radius (in $\tilde{r}$ ) in general than the fluctuations of $\phi^{\star}\left(\right.$ in $\left.r^{\star}\right)$. Thus we need to transform $\phi^{\star}$ such that the fluctuations of the transformed $\phi^{\star}$ reach their minimum at the same radius $\tilde{r}$ as those of $\phi$. This we achieve as follows. There is a uniform transformation $\Pi$ which translates all the points on the circle with radius $r^{\star}$ centered at the origin to the circle centered at the origin with radius $\tilde{r}$, the radius of $\phi$. If we apply $\Pi$ to $\phi^{\star}(r, \theta)$ then we get $\phi^{\star}\left(r+r^{\star}-\tilde{r}\right)$, the desired transformation of $\phi^{\star}(r, \theta)$. Now we can prove the next lemma:

Lemma A.2 For every $\epsilon>0$ holds:

$$
\left|\bar{f}_{N}^{\star}(r, \theta)\right| \leq\left|\bar{f}_{N}^{\star}\left(r+r^{\star}-\tilde{r}, \theta\right)\right|+\epsilon .
$$

The constant $r^{\star}$ is the radius of the circle parametrizing the set of mean-field solutions in the Ising case $(q=2)$. The constant $\tilde{r}$ is the radius $\hat{r}$ in the Potts case $q=3$ rescaled by the factor $2 / \sqrt{3}$, thus $\tilde{r}=(2 / \sqrt{3}) \hat{r}$.

Proof:

We use the following estimate, which is lemma 2.5 from $B \mathrm{BvEN}$ :

$$
\left|\mathbf{E} f_{N}^{\star}(r, \theta)-f_{N}^{\star}(r, \theta)\right| \leq \frac{\epsilon}{2} \text { a.e. on every bounded set. }
$$

Define:

$$
\mathcal{O}=\left\{\vec{z} \in \mathbf{R}^{2}:\|\vec{z}\|>r^{\star}+\delta\right\}, \mathcal{O}^{\prime}=\left\{\vec{z} \in \mathbf{R}^{2}:\|\vec{z}\|>\tilde{r}+\delta\right\} .
$$

Set $\mathcal{O} \subset \mathcal{O}^{\prime}$ because $\tilde{r} \leq r^{\star}$. Check this by using (7) and (8) and the scalingfactor $2 / \sqrt{3}$ for $\tilde{r}$. Decompose $\mathcal{O}^{\prime}$ as $\mathcal{O} \cup \mathcal{O}^{\prime} \backslash \mathcal{O}$. Because $\mathcal{O}^{\prime} \backslash \mathcal{O}$ is a finite set we can use estimate (14). With the already obtained estimate for $\mathcal{O}$ in [BvEN, (13) holds for all $(r, \theta) \in \mathcal{O}^{\prime}$. Because of (14) is true for all finite sets, (13) holds for all $(r, \theta)$.

Note that in a neighbourhood of $\tilde{r}$ it is equivalent to look in a neighbourhood of $r^{\star}$. Now we are able to prove the following theorem: 
Theorem A.1 Let $\mathcal{L}$ be the induced distribution of the overlap parameters and let $m=m(\theta)=(\tilde{r} \cos \theta, \tilde{r} \sin \theta)$, where $\theta \in[0, \pi)$ is a uniformly distributed random variable. Then:

$$
\mathcal{L}_{N, \beta} \stackrel{\mathcal{D}}{\rightarrow} \frac{1}{2} \delta_{m(\theta)}+\frac{1}{2} \delta_{-m(\theta)} \equiv \mathcal{L}_{\infty, \beta}[m] .
$$

Furthermore, the (induced) AW-metastate is the image of the uniform distribution of $\theta$ under the measure-valued map $\theta \rightarrow \mathcal{L}_{\infty, \beta}[m(\theta)]$.

First we prove the following two lemmas:

Lemma A.3 For $\phi_{N}$ and $\xi_{i}, \eta_{i}$, with $i \in \mathbf{N}$ as defined above, there exist strictly positive constants $W, W^{\prime}, l, l^{\prime}$ such that $(\tilde{r}$ is the largest solution of (8))

$$
\frac{\int_{|\|\vec{z}\|-\tilde{r}| \geq \delta_{N}} e^{-\beta N \phi_{N}(\vec{z})} d \vec{z}}{\int_{|\|\vec{z}\|-\tilde{r}|<\delta_{N}} e^{-\beta N \phi_{N}(\vec{z})} d \vec{z}} \leq W e^{-W N^{l}}
$$

on a set of $\mathbf{P}$-measure at least $1-W^{\prime} e^{-K^{\prime} N^{l^{\prime}}}$, where $\delta_{N}=N^{-\frac{1}{10}}$.

Lemma A.4 Assume the hypotheses of lemma A.3. Let $a_{N}=N^{-1 / 25}$. Then there exist strictly positive constants $K_{1}, K_{2}, C_{1}, C_{2}$ such that on a set of $\mathbf{P}$ measure at least $1-K_{1} e^{-N^{1 / 25}}$ the following bound holds,

$$
\frac{\int_{A_{N}^{\prime}} e^{-\beta N \phi_{N}(\vec{z})} d \vec{z}}{\int_{A_{N}} e^{-\beta N \phi_{N}(\vec{z})} d \vec{z}} \leq C_{1} e^{-N^{1 / 5}}
$$

where

$$
\begin{aligned}
& A_{N}=\left\{(r, \theta) \in \mathbf{R}_{0}^{+} \times[0,2 \pi)|| r-\tilde{r} \mid<\delta_{N}, g_{N}(\theta)-\min _{\theta} g_{N}(\theta)<a_{N}\right\} \\
& A_{N}^{\prime}=\left\{(r, \theta) \in \mathbf{R}_{0}^{+} \times[0,2 \pi)|| r-\tilde{r} \mid<\delta_{N}, g_{N}(\theta)-\min _{\theta} g_{N}(\theta) \geq a_{N}\right\} .
\end{aligned}
$$

Here

$g_{N}(\theta)=\frac{\sqrt{N}}{\beta} \mathbf{E}_{\psi} \mathbf{E}_{\zeta} \ln \left\{\frac{1}{2}+\cosh \beta \zeta \tilde{r} \cos \psi\right\}-\frac{1}{\beta \sqrt{N}} \sum_{i=1}^{N} \ln \left\{\frac{1}{2}+\cosh \left\{\beta \tilde{r} \zeta_{i} \cos \left(\theta-\psi_{i}\right)\right\}\right\}$,

which is the polar coordinate form of the function $g_{N}(\vec{z})$, which is defined as:

$$
g_{N}(\vec{z})=\frac{1}{\sqrt{N}} \sum_{i=1}^{N}\left\{\ln \left\{\frac{1}{2}+\cosh \beta \vec{z} \cdot(\xi, \eta)\right\}-\mathbf{E} \ln \left\{\frac{1}{2}+\cosh \beta \vec{z} \cdot(\xi, \eta)\right\}\right\} \text {. }
$$


It is convenient to look at the following decomposition:

$$
\begin{gathered}
\left(\phi_{N}-\mathbf{E} \phi_{N}\right)(\vec{z})=\beta \sqrt{N}\left(g_{N}\left(\vec{z}^{\prime}\right)+h_{N}(\vec{z})\right), \text { where } \\
h_{N}(\vec{z})=g_{N}(\vec{z})-g_{N}\left(\vec{z}^{\prime}\right) .
\end{gathered}
$$

The variable $\vec{z}^{\prime}$ is the projection of $\vec{z}$ onto $S^{1}(\tilde{r})$. Note that $\beta \sqrt{N} g_{N}=$ $\phi_{N}-\mathbf{E} \phi_{N} \equiv \bar{f}_{N}$. Define $g_{N}^{\star}$ and $h_{N}^{\star}$ in the same way but as decomposition of $\bar{f}_{N}^{\star}$ instead of $\bar{f}_{N}$.

Proof of lemma A.3:

Compare lemma 2.1 in $[\mathrm{BvEN}]$. Define:

$$
\begin{aligned}
& \mathcal{O}=\left\{\vec{z} \in \mathbf{R}^{2}:\|\vec{z}\|>r^{\star}+\delta\right\}, \mathcal{O}^{\prime}=\left\{\vec{z} \in \mathbf{R}^{2}:\|\vec{z}\|>\tilde{r}+\delta\right\}, \\
& \mathcal{I}=\left\{\vec{z} \in \mathbf{R}^{2}:\|\vec{z}\| \leq r^{\star}-\delta\right\}, \mathcal{I}^{\prime}=\left\{\vec{z} \in \mathbf{R}^{2}:\|\vec{z}\| \leq \tilde{r}-\delta\right\} .
\end{aligned}
$$

Now we first estimate the numerator which we can also write as:

$$
\int_{|| \vec{z} \|-\tilde{r} \mid \geq \delta_{N}} e^{-\beta N \phi_{N}(\vec{z})} d \vec{z}=\int_{\mathcal{O}^{\prime} \cup \mathcal{I}^{\prime}} e^{-\beta N \mathbf{E} \phi_{N}(\vec{z})} e^{-\beta N\left(\phi_{N}(\vec{z})-\mathbf{E} \phi_{N}(\vec{z})\right)} .
$$

With lemma A.2 we have the following inequality:

$$
\begin{gathered}
\sup _{\vec{z} \in \mathcal{O}^{\prime}}\left|\bar{f}_{N}^{\star}(r, \theta)\right|-\epsilon \leq \sup _{\vec{z} \in \mathcal{O}^{\prime}}\left|\bar{f}_{N}^{\star}\left(r+r^{\star}-\tilde{r}, \theta\right)\right|=\sup _{\vec{z} \in \mathcal{O}}\left|\bar{f}_{N}^{\star}(r)\right| \\
\mathbf{P}\left[\sup _{(r, \theta) \in \mathcal{O}^{\prime}}\left|\bar{f}_{N}^{\star}(r, \theta)\right|-\epsilon \geq \frac{C}{2}(r-\tilde{r})^{2}\right] \leq \mathbf{P}\left[\sup _{(r, \theta) \in \mathcal{O}}\left|\bar{f}_{N}^{\star}(r, \theta)\right| \geq \frac{C}{2}\left(r-r^{\star}\right)^{2}\right] .
\end{gathered}
$$

Lemma 2.4 of BvEN tells us that this event is of measure zero. Now we can estimate the integral.

First we estimate $\mathbf{E} \phi_{N}^{\star}(\vec{z})$. Because $\mathbf{E} \phi_{N}^{\star}(\vec{z})$ is a bounded function, in each bounded interval one can always bound it from below by a function of the following kind:

$$
\mathbf{E} \phi_{N}^{\star}(\vec{z}) \geq C(\|\vec{z}\|-\tilde{r})^{2}+\mathbf{E} \phi_{N}^{\star}(\tilde{r}), \text { with } C \text { a positive bounded constant. }
$$

Then

$$
\mathbf{E} \phi_{N}^{\star}\left(\vec{z}+r^{\star}-\tilde{r}\right) \geq C\left(\|\vec{z}\|-r^{\star}\right)^{2}+\mathbf{E} \phi_{N}\left(r^{\star}\right)
$$


when we apply $\Pi$ to this estimate. Now use estimate (15) with this constant $C$. Then it holds:

$$
\begin{gathered}
\int_{\mathcal{O}^{\prime}} e^{-\beta N \mathbf{E} \phi_{N}(\vec{z})} e^{-\beta N\left(\phi_{N}(\vec{z})-\mathbf{E} \phi_{N}(\vec{z})\right)} d z \leq \int_{\mathcal{O}} e^{-\beta N \mathbf{E} \phi_{N}^{\star}\left(\vec{z}+r^{\star}-\tilde{r}\right)} e^{\beta N\left|\bar{f}_{N}^{\star}(\vec{z})\right|} e^{\epsilon \beta N} d \vec{z} \leq \\
e^{-\beta N\left(\mathbf{E} \phi_{N}^{\star}\left(r^{\star}\right)-\epsilon\right)} \int_{\mathcal{O}} e^{-\beta N C\left(r-r^{\star}\right)^{2}} e^{\beta N \frac{C}{2}\left(r-r^{\star}\right)^{2}} d r=e^{\beta N\left(\mathbf{E} \phi_{N}^{\star}\left(r^{\star}\right)-\epsilon\right)} \int_{\mathcal{O}} e^{-\beta N \frac{C}{2}\left(r-r^{\star}\right)^{2}} d r \leq \\
\cdots \leq 2 \pi \frac{2}{\beta N C} \exp \left(-\beta N\left(\mathbf{E} \phi_{N}^{\star}\left(r^{\star}\right)-\epsilon\right)\right) \exp -\beta N C_{2}\left(\frac{\delta^{2}}{4}\right) .
\end{gathered}
$$

For further details see $[\mathrm{BvEN}$. The interior $\mathcal{I}$ gives a similar expression. Notice that the image of $\mathcal{I}$ under the transformation $\Pi: r \rightarrow r+r^{\star}-\tilde{r}$ is $\mathcal{I} \backslash B\left(0, r^{\star}-\tilde{r}\right)$. The ball $B\left(0, r^{\star}-\tilde{r}\right)$ is a finite set so we can integrate over $\mathcal{I}$ instead of $\mathcal{I} \backslash B\left(0, r^{\star}-\tilde{r}\right)$ by (14). To estimate the denominator we just replace $r^{\star}$ by $\tilde{r}$ in the expressions of the proof of lemma 2.1 in [BvEN, pag.192,193]. Combining the estimates for the numerator and the denominator gives the desired result.

Proof of lemma A.4:

¿From this moment we ignore the constants which enter by applying lemma A.1, because they cancel out when we divide the numerator by the denominator. For $\left|h_{N}\right|$ it holds:

$$
\left|h_{N}\right| \leq\left|h_{N}^{\star}\right| \leq \epsilon
$$

by lemma 2.6 of BvEN]. Consider the following integral:

$$
\int_{\theta: g_{N}(\theta)>a_{N}+\min _{\theta} g_{N}(\theta)} e^{-\sqrt{N} g_{N}(\theta)} \leq 2 \pi e^{-\sqrt{N} \min _{\theta} g_{N}(\theta)} e^{\sqrt{N} a_{N}} .
$$

Henceforth it is just a matter of plugging in to get the desired estimate on the denominator. We refer to [BvEN] for the details. One gets a estimate for the denominator in the same way. By dividing the two estimates lemma A.4 is proven.

Proof of theorem A.1:

In the preceding paragraphs we have seen that the measures $\tilde{\mathcal{L}}$ concentrate on a circle at the places where the random function $g_{N}(\theta)$ takes its minimum. Now it only remains to show that these sets degenerate to a single point, a.s. 
in the limit $N \rightarrow \infty$. If we have proven it for $\tilde{\mathcal{L}}$, then we have proven it also for $\mathcal{L}$, because $\lim _{N \rightarrow \infty} \tilde{\mathcal{L}}=\mathcal{L}$. With the help of [BvEN] this is very easy, because we can use Proposition 3.4 with the function

$$
g(.)=\ln \left\{\cosh \beta .+\frac{1}{2}\right\} .
$$

This works because $g$ is an aperiodic even function. And of course Proposition 3.7 also holds for this $g$. These two propositions we use, tell us that the process $\eta_{N}=g_{N}(\theta)-\mathbf{E} g_{N}(\theta)$ converges to a strictly stationary Gaussian process, having a.s. continuously differentiable sample paths. And on any interval $[s, s+t], t<\pi$ the function $\eta_{N}$ has only one global minimum. Furthermore, if we define the sets:

$$
L_{N}=\left\{\theta \in[0, \pi): \eta_{N}(\theta)-\min _{\theta^{\prime}} \eta_{N}\left(\theta^{\prime}\right) \leq \epsilon_{N}\right\},
$$

with $\epsilon_{N}$ some sequence converging to zero, $L_{N} \stackrel{\mathcal{D}}{\rightarrow} \theta^{\star}$. Then the remarks below Proof of theorem 3 in [BvEN] conclude the proof.

This research was supported by the Samenwerkingsverband Mathematische Fysica. A.v.E. thanks Christian Borgs for asking the question how the results of $\mathrm{BvEN}$ generalize to Potts spins.

\section{References}

[vEHP] A.C.D. van Enter, J.L. van Hemmen, and C. Pospiech, Mean-field theory of random-site q-state Potts models, J. Phys. A 21, 791-801 (1988).

[BvEN] A. Bovier A.C.D. van Enter, B. Niederhauser, Stochastic symmetry-breaking in a Gaussian Hopfield model, J. Stat. Phys. 95, 181-213 (1999).

[B] A. Bovier, Statistical mechanics of disordered systems, MaPhySto Lecture Notes 10, Aarhus (2001).

[BG] A. Bovier, V. Gayrard, Hopfield models as generalized random mean field models, in Mathematical Aspects of spin glasses and neural networks, Progress in Probability 41, Birkhäuser, Boston, (1997). 
[G] V. Gayrard, Thermodynamic limit of the q-state Potts-Hopfield model with infinitely many patterns, J. Stat. Phys. 68, 977-1011 (1992).

[HvEC] J.L. van Hemmen, A.C.D. van Enter, and J. Canisius, On a classical spin glass model, Z. Phys. B - Condensed matter 50, 311-336 (1983).

[MPV] M. Mézard, G. Parisi and M.'A. Virasoro, Spin Glass Theory and Beyond, World scientific, (1987).

[Nie] B. Niederhauser, Mathematical Aspects of Hopfield Models, Dissertation TU-Berlin, 2000.

[NS] C.M. Newman and D.L. Stein, Thermodynamic Chaos and the structure of short-range spin glasses, in Mathematical Aspects of spin glasses and neural networks, Progress in Probability 41, Birkhäuser, Boston, (1997).

[NS2] C.M. Newman and D.L. Stein, The State(s) of Replica Symmetry Breaking: Mean Field Theories vs Short-Ranged Spin Glasses, formerly known as Replica Symmetry Breaking's New Clothes, J. Stat.Phys. to appear, cond-mat/0105282 (2001).

[Wu] F.Y. Wu, The Potts-model, Rev. Mod. Phys. 54, 235 (1982). 